\title{
Kidney organoids: current knowledge and future directions
}

\author{
Niloofar Khoshdel-Rad ${ }^{1,2} \cdot$ Amin Ahmadi $^{1,3} \cdot$ Reza Moghadasali $^{1,2}(\mathbb{D}$ \\ Received: 9 June 2021 / Accepted: 14 December 2021 / Published online: 28 January 2022 \\ (c) The Author(s), under exclusive licence to Springer-Verlag GmbH Germany, part of Springer Nature 2021
}

\begin{abstract}
The kidney is a highly complex organ in the human body. Although creating an in vitro model of the human kidney is challenging, tremendous advances have been made in recent years. Kidney organoids are in vitro kidney models that are generated from stem cells in three-dimensional (3D) cultures. They exhibit remarkable degree of similarities with the native tissue in terms of cell type, morphology, and function. The establishment of 3D kidney organoids facilitates a mechanistic study of cell communications, and these organoids can be used for drug screening, disease modeling, and regenerative medicine applications. This review discusses the cellular complexity during in vitro kidney generation. We intend to highlight recent progress in kidney organoids and the applications of these relatively new technologies.
\end{abstract}

Keywords 3D culture $\cdot$ In vitro models $\cdot$ Kidney development $\cdot$ Kidney organoids $\cdot$ Organogenesis

\begin{tabular}{|c|c|c|c|c|}
\hline \multicolumn{3}{|c|}{ Abbreviations } & hrsACE2 & Human recombinant soluble ACE2 \\
\hline \multicolumn{2}{|c|}{$3 \mathrm{D}$} & Three-dimensional & IM & Intermediate mesoderm \\
\hline \multicolumn{2}{|c|}{ AIM } & Anterior intermediate mesoderm & iPSCs & Induced pluripotent stem cells \\
\hline \multicolumn{2}{|c|}{ AKI } & Acute kidney injury & KDR/VEGFR2/Flk1 & Kinase insert domain protein \\
\hline \multicolumn{2}{|c|}{ BMP } & Bone morphogenetic protein & & receptor \\
\hline \multicolumn{2}{|c|}{ CAM } & Chorioallantoic membrane & K-ECM & Kidney-derived extracellular \\
\hline \multicolumn{2}{|c|}{$\mathrm{CF}$} & Cystic fibrosis & & matrix \\
\hline \multicolumn{2}{|c|}{$\mathrm{ECM}$} & Extracellular matrix & LTL & Lotus tetragonolobus lectin \\
\hline \multicolumn{2}{|c|}{$\mathrm{ECs}$} & Endothelial cells & c-MET & Tyrosine-protein kinase Met \\
\hline \multicolumn{2}{|c|}{ FGF } & Fibroblast growth factor & & (mesenchymal-epithelial-transition) \\
\hline \multirow{2}{*}{\multicolumn{2}{|c|}{ FOX }} & Forkhead/winged helix transcrip- & $\mathrm{MM}$ & Metanephric mesenchyme \\
\hline & & tion factor & MSCs & Mesenchymal stem cells \\
\hline \multirow{2}{*}{\multicolumn{2}{|c|}{ GATA }} & Trans-acting T cell-specific tran- & NPCs & Nephron progenitor cells \\
\hline & & scription factor & Odd1 or OSR 1 & Odd-skipped related 1 \\
\hline \multicolumn{2}{|c|}{ GDNF } & Glial cell-derived neurotrophic & PAX & Paired box protein \\
\hline \multirow{2}{*}{\multicolumn{2}{|c|}{ GGT }} & factor & PDGFR & Platelet-derived growth factor \\
\hline & & Gamma-glutamyl transpeptidase & & receptor \\
\hline \multicolumn{2}{|c|}{ hPSCs } & Human pluripotent stem cells & PECAM-1/CD31 & $\begin{array}{l}\text { Platelet/endothelial cell adhesion } \\
\text { molecule-1 }\end{array}$ \\
\hline \multirow[t]{3}{*}{$\square$} & \multirow{3}{*}{\multicolumn{2}{|c|}{$\begin{array}{l}\text { Reza Moghadasali } \\
\text { rezamoghadasali@yahoo.com; } \\
\text { rezamoghadasali@ royaninstitute.org }\end{array}$}} & PIM & Posterior intermediate mesoderm \\
\hline & & & PKD & Polycystic kidney disease \\
\hline & & & PRCC & Papillary renal cell carcinoma \\
\hline \multirow[t]{3}{*}{1} & \multirow{3}{*}{\multicolumn{2}{|c|}{$\begin{array}{l}\text { Department of Stem Cells and Developmental Biology, } \\
\text { Cell Science Research Center, Royan Institute for Stem Cell } \\
\text { Biology and Technology, ACECR, Tehran, Iran }\end{array}$}} & $\mathrm{RCC}$ & Renal cell carcinoma \\
\hline & & & SLC & Solute carrier \\
\hline & & & SIX2 & Sine oculis-related homeobox 2 \\
\hline \multirow[t]{2}{*}{2} & \multirow{2}{*}{\multicolumn{2}{|c|}{$\begin{array}{l}\text { Department of Developmental Biology, University of Science } \\
\text { and Culture, Tehran, Iran }\end{array}$}} & UB & Ureteric bud \\
\hline & & & UBPCs & Ureteric bud progenitor cells \\
\hline & \multicolumn{2}{|c|}{ Research Center fo } & VEGF & Vascular endothelial growth factor \\
\hline
\end{tabular}

Biomedicine Institute, Tabriz University of Medical

Sciences, Tabriz, Iran 
WT1

WNT

Wilms tumor 1

Wingless-type mouse mammary

tumor virus integration site

\section{Introduction}

The kidney is one of the most complex and highly organized organs in the human body. This organ contains approximately one million functional units called nephrons that are responsible for blood filtering. Each nephron, which consists of various specialized cell types, comprises a renal corpuscle connected to complicated and convoluted tubules that drain into a collecting duct (Liao et al. 2020). The number of nephrons may vary according to age and health status, but their numbers at birth are determined during kidney organogenesis. Kidney diseases are associated with irreversible loss of nephrons. Therefore, in addition to drug therapy, there is a fundamental need to develop new therapeutic approaches (Schnaper 2014). Although some progress has been made in the last two decades, in vitro recapitulation of kidney structures remains challenging. Primary and immortalized human cell lines have enabled detailed research on specific kidney cell types, but these cells lack the histological features of the kidney. Besides, the immortalization process interferes with the evaluation of the cell cycle and related diseases. On the other hand, experiments in animals offer better physiological models; however, they are less than ideal because of interspecific differences and ethical concerns (Faria et al. 2019).

The lack of human models for the kidneys hampers investigations into their physiology and pathophysiology. Advanced in vitro models are needed to have a better understanding of kidney development and facilitate the improvement of precision medicine. Recently, many studies have been performed with the intent to produce in vitro three-dimensional (3D) models of functional tissues and organs. These 3D in vitro models provide better conditions for approaching native physiological conditions when compared with the conventional two-dimensional (2D) models because they form structures that have specific polarity and increasing cellular communication (Kang et al. 2019). In recent years, the generation of kidney organoids has opened up new approaches for research on kidney development, drug screening, and disease modeling. This review discusses the cellular complexity during kidney development and how our knowledge about kidney development leads to the generation of kidney organoids in the laboratory. Finally, we discuss existing approaches to increasing the complexity of kidney organoids, as well as the potential applications for these organoids in disease

modeling, drug screening, personalized medicine, and regenerative medicine.

\section{Cellular complexity during in vitro kidney generation}

The human kidney is a complex organ that encompasses higher-order structures and multiple specialized cell types; therefore, it is difficult to generate an appropriate model to study kidney development and disease. In vitro kidney structures can be created following the same developmental process that occurs during the embryonic period (Chow et al. 2020). During this developmental period, the metanephros kidney differentiates as a result of a bidirectional inductive interaction between the ureteric bud (UB) lineage, which is derived from a more anterior intermediate mesoderm (AIM), and the metanephric mesenchyme (MM), which is derived from the most posterior intermediate mesoderm (PIM) (Taguchi et al. 2014). Early morphogenetic events during kidney organogenesis lead to the formation of multiple distinct renal progenitor populations. The renal nephrogenic niche includes at least four major self-renewing, multipotent progenitor cell populations: ureteric bud progenitor cells (UBPCs), nephron progenitor cells (NPCs), endothelial progenitor cells (EPCs), and stromal progenitors (Mugford et al. 2008; Khoshdel Rad et al. 2020). Furthermore, kidney organogenesis is dependent on the migration of stromal and endothelial cells (ECs) from other embryonic regions into the developing kidney (Bronner-Fraser and Fraser 1988; Schmidt-ott et al. 1993; Guillaume et al. 2009). Precise multicellular patterning and spatiotemporal signaling between several distinct cell populations within the nephrogenic niche lead to ongoing nephrogenesis and generation of a vascularized kidney with a complex structure (Khoshdel Rad et al. 2020). Next, we will discuss differentiation of these cell populations during embryogenesis and in vitro organogenesis.

UB precursors undergo terminal differentiation and a highly stereotypical set of morphological changes to form the collecting duct system (Al-Awqati 2013; Sinha et al. 2017). Unlike the developing human kidney, most current kidney differentiation protocols do not have any evidence for a branching ureteric epithelium. These kidney structures lack a connection between the collecting duct and distal segments of nephrons (Little and Combes 2019). NPCs possess the unique capability for a self-renewal state and nephrogenic potential to maintain the nephron progenitor pool and generate all cell types of a nephron. NPCs acquire an epithelial-like morphology. Thereafter, they undergo morphological changes to form pre-tubular aggregates that develop into renal vesicles, comma- and S-shaped bodies, and mature nephrons. One nephron is composed of more 
than 20 various cell types, including a podocyte, proximal tubule (convoluted and straight components), the loop of Henle, a distal tubule, and collecting tubule cells (Al-Awqati and Oliver 2002). Although most current kidney differentiation protocols generate early patterning and segmenting nephrons, generation of mature functional epithelium is a major challenge (Wu et al. 2018). Some scientists believe that it is impossible to simultaneously generate both the MM and the UB, and they use a distinct protocol to generate collecting ducts and nephrons (Taguchi et al. 2014; Taguchi and Nishinakamura 2017).

Kidney tissues are maintained by several supportive cells of the stromal and endothelial lineages, and failure of these supportive cells results in renal dysfunction. Both stromal and ECs have critical roles in proper kidney development (Mugford et al. 2008). The renal interstitial stroma is a heterogeneous cell population that acts as physical support for developing cells, and it is a source of specialized cells that secret the extracellular matrix (ECM) and paracrine factors. Stromal progenitors are located adjacent to the anterior part of NPCs in the MM and later encompass both UBs and nephrons. Stromal cells have critical roles in UB outgrowth and NPC differentiation. Most renal stromal cells originate from odd-skipped related 1 (OSR1)-positive multipotent precursors in the PIM (Mugford et al. 2008). Groups of stromal cells that migrate from other embryonic tissues include the neural crest and paraxial mesoderm, which integrate into the nascent stromal compartment of the developing kidney (Bronner-Fraser and Fraser 1988; Guillaume et al. 2009). Furthermore, T-box 18 (TBX18)-positive multipotent progenitors migrate into the metanephric kidney and give rise to the ureteric mesenchyme and renal interstitial cells (Bohnenpoll et al. 2013). Stromal progenitors give rise to various cell types, including mural cells (vascular smooth muscle cells and pericytes), mesangial cells, interstitial fibroblasts, and renin-producing cells (Kobayashi et al. 2014). Current methods for pluripotent stem cell (PSC) differentiation into renal lineage produce several renal stromal populations and other related cells (Wu et al. 2018); however, these cells are not patterned likewise in an in vivo organ. A possible explanation for this problem is that several groups of stromal cells migrate from external embryonic tissues into the metanephric kidney. Thus, some stromal populations are not generated in current differentiation methods and their cellular interactions are lost (Little and Combes 2019). Blood vessels transport oxygen and nutrients in embryonic and adult kidneys. ECs also play a key role in the development of neighboring cells in the nephrogenic niche, which promotes nephrogenesis (Munro et al. 2017). Several EPC populations play a key role in the formation of de novo vessels and nephrogenesis during kidney development (Munro et al. 2017). There is extremely limited generation of ECs in the majority of current differentiation methods ( $0.3 \%$ or less of total cells) (Wu et al. 2018; Khoshdel-rad et al. 2021); many of these ECs have immature phenotypic characteristics. In addition, glomerular capillary formation is rare (Czerniecki et al. 2018). The results of studies show that transplantation of human pluripotent stem cell (hPSC)-derived renal cells into highly vascularized sites such as the chorioallantoic membrane (CAM) (Garreta et al. 2019), mouse lymph node (Francipane and Lagasse 2015), and subrenal capsule (Bantounas et al. 2018; Koning et al. 2018; Tanigawa et al. 2018; van den Berg et al. 2018) improved vascularization and maturation of the renal cells. Lack of vascularization could potentially be overcome by in vitro addition of ECs (Takebe et al. 2015; Khoshdel-rad et al. 2021) and incorporation of fluid shear stress (Homan et al. 2019). Although some progress has been made in recent years, in vitro vascularization of kidney structures remains a challenge.

\section{Kidney organogenesis in a dish}

Some cell lines permit detailed research on specific kidney cell types. Primary and immortalized human cell lines have been used to study kidney physiology and disease with some success, but these cells lack the histological features of the kidneys. Primary renal cells have limited proliferation potential and lose their phenotypic characteristics over time. Although virally transformed immortalized cell lines proliferate indefinitely, the immortalization process may alter their characteristics and function and interfere with the evaluation of the cell cycle and related diseases (Faria et al. 2019). These 2D models are unable to mimic the cellular interactions and microenvironmental signals within complex heterogeneous tissues. Cell polarity and complex cellular communications are absent in 2D culture models (Kang et al. 2019). Because of the drawbacks of 2D cellular models, researchers are seeking alternative 3D model systems. The 3D models with multicellular constructs and their specific ECM are more complex and have more differentiated cells and better physiological functionality compared to their simple 2D counterparts. These 3D models can be created using different cells and methods, and they provide a valuable research model for studying organ development and its functions (Kang et al. 2019). Studies show that the use of a longer 3D culture leads to the formation of more mature structures by increasing the cell-to-cell and cell-toECM interactions. Thus, 3D culture methods are a better way to provide relevant environmental support and signaling cues (Garreta et al. 2019).

Creating a 3D in vitro model of the human kidney is challenging; however, tremendous advances have been made in 
recent years. Kidney organoids are in vitro kidney models that generate from (stem) cells under 3D culture conditions. They exhibit remarkable degrees of similarity with the native tissue in terms of cell type, morphology, and function (Gupta et al. 2020). Kidney organoid technology may rely on generation of 3D kidney structures from adult stem cells (ASCs) (Schutgens et al. 2019) or PSCs (Morizane et al. 2015; Takasato et al. 2015; Garreta et al. 2019). In the following sections, we describe studies of kidney organoids derived from tissue-resident cells and PSCs.

\section{Adult stem cell-derived kidney organoids}

ASC-derived organoids are 3D epithelial structures that represent the major features of the original tissue (Schutgens et al. 2019). These organoids are suitable models for studying adult tissue regeneration processes. ASC-derived kidney organoids have less complexity than PSC-derived kidney organoids; they contain only renal epithelial cells and lack nerves, blood vessels, and stromal structures. Clevers et al. isolated primary renal epithelial cells from either urine or cortical kidney tissue. These cells were seeded in Matrigel and cultured in growth factor-rich medium. Kidney tubuloids with cystic structures were obtained within 6 days after seeding. These tubuloids contained simple cuboidal epithelial cells and could mimic the regenerative response of an adult kidney. The kidney tubuloids contained only the epithelial cells of the proximal tubule, loop of Henle, distal tubule, and collecting duct segments, and they lacked important components such as stromal and ECs. These organoids could be grown and maintained for up to 20 passages. ASC-derived tubuloids were used as a model for further investigation of infectious, malignant, and hereditary kidney diseases (Schutgens et al. 2019). Another research group isolated urine-derived stem cells (USCs) from fresh urine samples, and these stem cells had self-renewal and multipotent differentiation potential. The USCs were differentiated into renal tubular epithelial cells using solubilized porcine kidney-derived extracellular matrix (K-ECM). After 14 days, 3D human renal organoids that expressed glomerular and proximal tubule markers were formed (Guo et al. 2020). The heterogeneity of urine-derived cells used to produce organoids could be mentioned as a limitation for these kinds of kidney organoids. These organoids lacked stromal and ECs. Furthermore, the number of proximal cells was higher than the other types of renal tubular cells. However, these organoids provide a rapid, cost-effective approach for producing a suitable in vitro model because they are obtained by tissue biopsy or the patient's urine (Schutgens et al. 2019).

In another study, Ding and colleagues established a 3D in vitro human renal organoid generated from human kidney fragments. Human renal cells were isolated from the donor's kidneys and successfully seeded onto a hanging drop plate. Cell aggregates were cultured in a kidney culture medium supplemented with solubilized human K-ECM. These 3D multicellular organoids contained some of the renal cell types. The study results showed that this model could be used for drug screening and nephrotoxicity assays (Ding et al. 2020). Zeng and colleagues established an expandable, branching UB organoid that could be generated from primary UBPCs from kidney tissues or developed de novo from hPSCs. The authors isolated primary UBPCs from mouse (E11.5) and human (9-13-week) fetal kidneys and seeded them into U-bottom 96-well low-attachment plates. After $24 \mathrm{~h}, \mathrm{UB}$ cell aggregates were formed and they were embedded into Matrigel droplets. After around 10-15 days, UB tip structures branched out from the aggregates. These structures were dissected out and re-embedded into Matrigel and extended as intact T-shaped UB organoids. Thereafter, the medium was replaced with a collecting duct differentiation medium, which resulted in collecting duct organoid formation. During the final differentiation phase, the expression of UBPC markers was greatly decreased, while principal and intercalated-specific markers were dramatically elevated (Zeng et al. 2021). Future studies on organoids derived from tissue-resident stem cells should focus on optimizing the culture medium to create all parts of the kidney (Schutgens et al. 2019). Autologous ASC-derived organoids could enforce personalized patient-derived 3D models with higher sophisticated phenotypes. Table 1 summarizes the differentiation protocols and terminally differentiated cells in the ASC-derived organoid structures from different studies.

\section{Human pluripotent stem cell-derived kidney organoids}

The ability to direct differentiation of hPSCs towards renal lineages has provided an unlimited source of renal cell types, which would overcome both the poor availability and the accessibility of primary human samples (Zahmatkesh et al. 2021). Over the past decade, developmental biology has guided the design of kidney organoid differentiation protocols. A combination of small molecules and growth factors is essential for differentiation into a renal lineage (Morizane et al. 2015). The most important studies in the field of kidney organoids were published in 2015. These studies were performed separately at Bonventre's laboratory (Morizane and colleagues differentiation protocol) and Little's laboratory. These researchers differentiated hPSCs into kidney organoids (Morizane et al. 2015; Takasato et al. 2015). Other studies recently used biological and engineering approaches to improve the maturation of kidney organoids. A protocol by Little's laboratory could generate human-induced pluripotent stem cell (hiPSC)-derived kidney organoids that contain more than 500 nephrons with renal-specific cell populations such as podocytes, proximal and distal tubular cells, renal stromal cells (cortical and medullary), an endothelial 


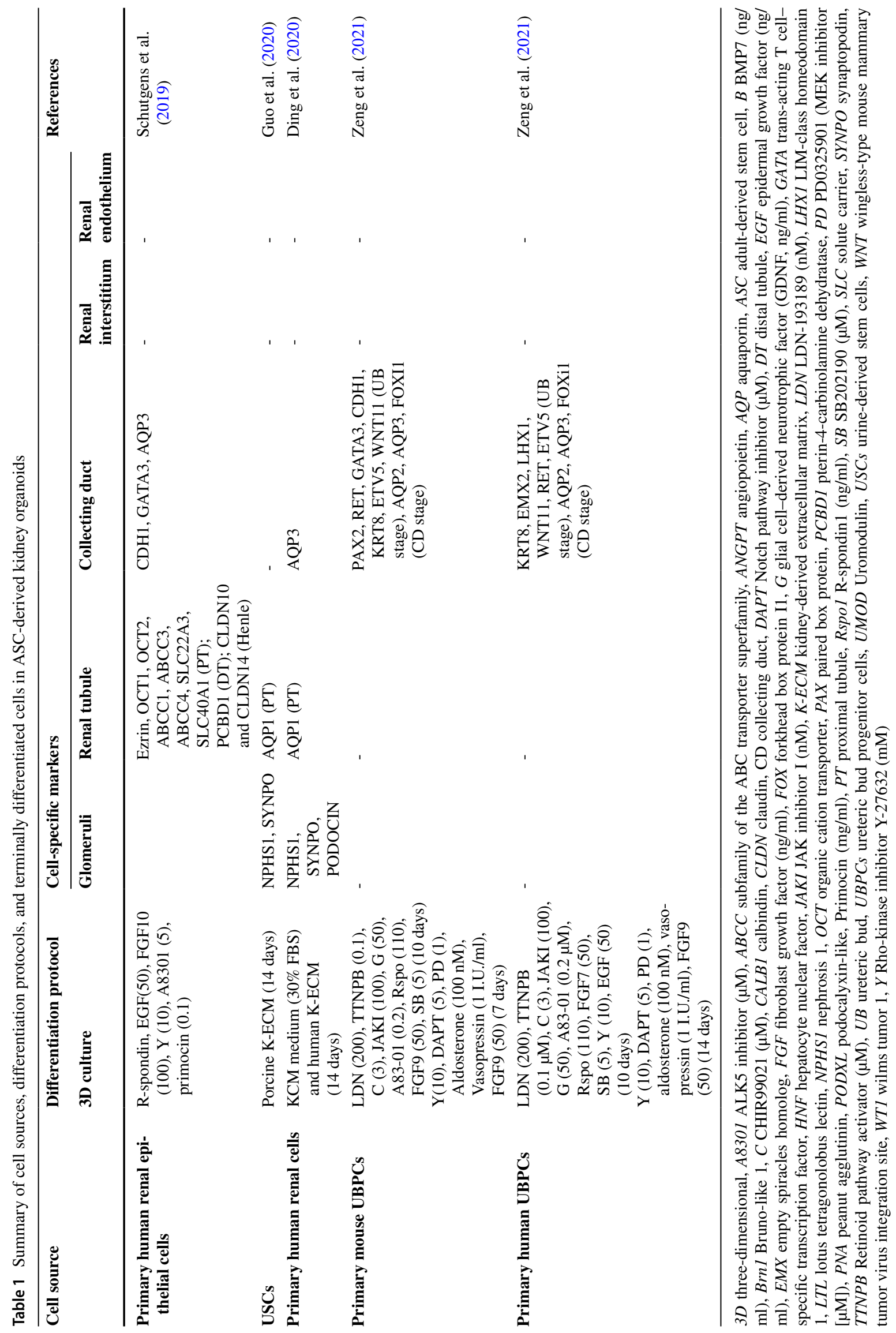


network, and various components of the renin-angiotensin system (Takasato et al. 2015). Their kidney organoids had $21 \%$ non-renal cells (Wu et al. 2018). RNA sequencing analysis showed that these organoids resembled the first trimester of fetal kidney tissue. Several studies utilized this protocol to conduct in vivo transplantations (Koning et al. 2018), developmental research (Bantounas et al. 2018), renal disease modeling (Forbes et al. 2018; Howden et al. 2018), scale-up of organoid generation (Kumar et al. 2019), encapsulating kidney organoids (Geuens et al. 2021), and bioprinting technology (Lawlor et al. 2021) and to study the role of the renin-angiotensin system (Shankar et al. 2021). Bonventre's laboratory created a protocol that generated NPCs with 75-92\% efficiency in 9 days. They used lower doses of fibroblast growth factor (FGF) $9(10 \mathrm{ng} / \mathrm{ml})$ than the protocol by Little's laboratory. Their 3D kidney organoids contained multiple kidney compartments such as nephron segments, a collecting duct, and endothelial and interstitial cells. (Morizane et al. 2015). These organoids contained only $11 \%$ non-renal cells (Wu et al. 2018).

Garreta and colleagues promoted the speed and efficiency of maturation by applying defined renal inductive signals (CHIR99021, Activin A, and FGF9) and a proper substrate. After 16 days, there were hPSC-derived kidney organoids that contained proximal tubules, loops of Henle, distal tubules, and glomeruli. These researchers reported that a soft microenvironment increased the expressions of mesodermal and renal-specific genes (Garreta et al. 2019). In another study, Low and colleagues established a versatile approach that used a three-step CHIR99021 treatment to generate patterned and vascularized hPSC-derived kidney organoids. However, these organoids lacked a loop of Henle and collecting duct. Modulation of wingless-type mouse mammary tumor virus integration site (WNT) signaling regulates the relative proportion of proximal versus distal nephron segments within kidney organoids. Single-cell analysis identified a population of $\mathrm{SIX} 1^{+} /$kinase insert domain protein receptor $(\mathrm{KDR})^{+} \mathrm{NPC}$ as a potential source of de novo vessel formation that formed from day 11. Vascular endothelial growth factor (VEGF)-A secretion by the developing podocytes supported further maturation of the EPCs. Structural maturation of these organoids occurred after transplantation beneath the renal capsules of NSG (NOD.Cg-Prkdcscid. Il 2 rgtm $1 \mathrm{Wjl} / \mathrm{SzJ}$ ) mice. These authors provided the first evidence that, in the absence of exogenous angiogenic factors, a de novo vascular network in hPSC-derived kidney structures could be established (Low et al. 2019).

Most studies of kidney organoids relied on the generation of 3D nephron structures, but not the contiguous epithelial network of the kidneys' collecting ducts. Zeng et al. established a stepwise method that generated hPSC-derived branching UB organoids. These UB organoids could be stably passaged every 6-10 days, depending on their size. The
UB organoids underwent maturation to induce collecting duct organoids, which expressed principal (AQP2, AQP3, and AQP4) and intercalated (Forkhead/winged helix transcription factor I1 (FOXI1)) markers (Zeng et al. 2021). The abovementioned studies all generated hPSC-derived kidney organoids by using growth factors and small molecules. In one study, Hiratsuka et al. showed that hPSCs could be converted into $3 \mathrm{D}$ kidney structures by using only syn-mRNA encoding transcription factors in the absence of growth factors. These researchers developed a different strategy that used two sets of synthetic mRNAs for stepwise transfection of hPSCs into nephron-like organoids in a suspension culture. After 14 days, nephron-like tissues composed of proximal tubules, distal tubules, and podocytes were generated. However, some segment-specific markers (such as CRB2, HNF1B, and KCNJ1) showed less expression compared to kidney organoids induced by growth factors (Hiratsuka et al. 2019).

Table 2 summarizes the PSC lines, differentiation protocols, and terminally differentiated cells in the PSC-derived organoids of several studies. There is tremendous variation between the differentiation experiments, which may be due to technical approaches that underlie the generation of kidney organoids, such as inter-reagent and batch-to-batch variability, variations between different lines of PSCs, and the skill of the technician. Kidney organoids generated from hPSCs have many unresolved challenges that include immature renal cells, a nascent vascular system, lack of a properly developed glomerular basement membrane (GBM), lack of interconnection between nephrons and collecting ducts in organoid structures, and tumorigenic risk (Howden et al. 2018; Miyoshi et al. 2019). Therefore, using bioengineering approaches to create structures similar to the original tissue is essential for reproducible and large-scale organoid generation.

\section{Current approaches in the generation of more complex organoids}

Recent studies have logically shifted toward using biological and bioengineering approaches to better resemble the native kidney tissue in an attempt to improve kidney organoid complexity. In the following section, we describe current approaches and future research directions for the generation of higher-order and vascularized kidney organoids (Fig. 1).

\section{Co-culture systems}

Recent developments of co-culture systems within the organoid technology provide a novel approach for investigating cellular interactions. Supportive co-culture conditions that include various types of endothelial and stromal cells 


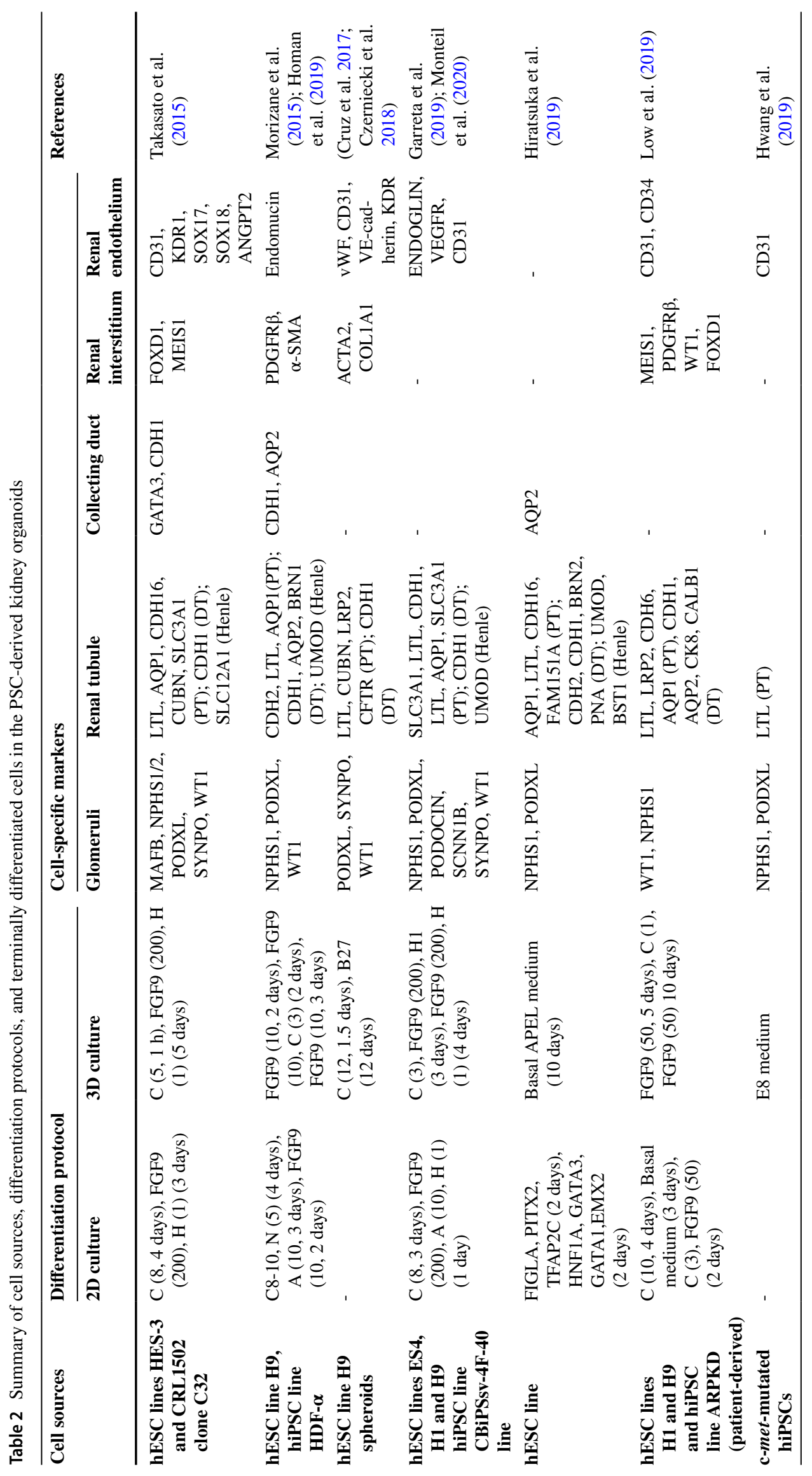




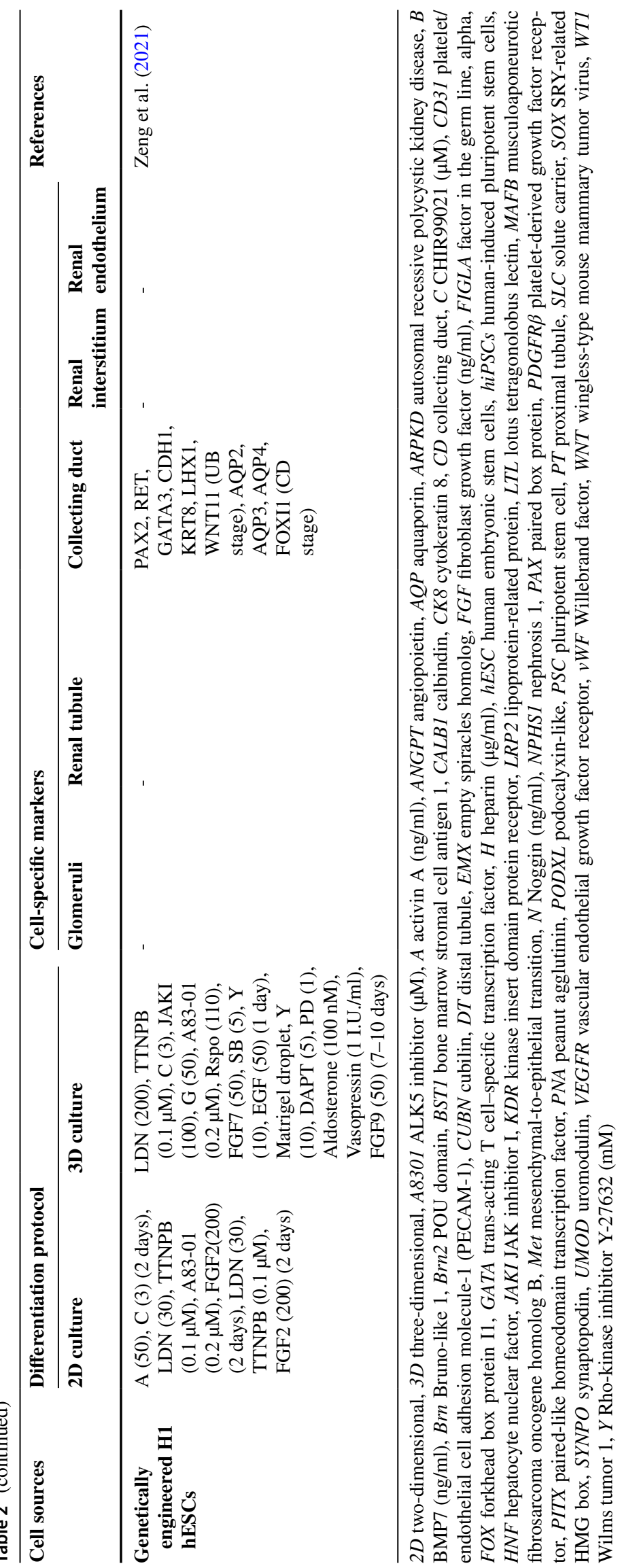




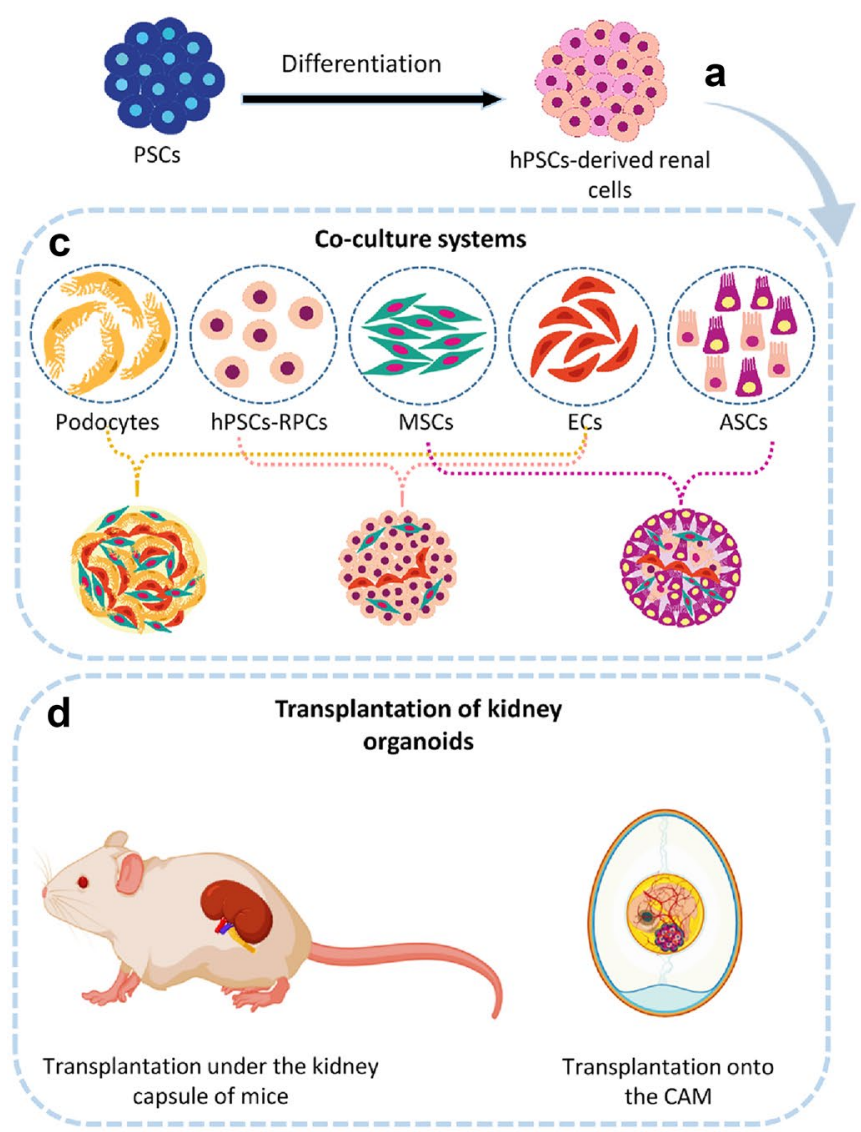

Fig. 1 Overview of the main current approaches for generation of higher complex kidney organoids. Kidney organoid technology relies on the generation of three-dimensional (3D) kidney structures from (A) pluripotent stem cells (PSCs), as embryonic stem cells and induced pluripotent stem cells (iPSCs); (B) renal tissue-derived

provide the proper microenvironmental niche, facilitate organoid assembly, and improve organoid vascularization and maturation (Zahmatkesh et al. 2021). There is strong evidence that different types of stromal cells could improve vascularization and maturation of 3D kidney constructs by secretion of cytokines and growth factors. Taguchi and Nishinakamura established an organoid co-culture system composed of three major renal precursors. They developed methods for differential induction of mouse pluripotent stem cells (mPSCs) into nephron and UBPCs with distinct cues and co-cultured these cells with primary platelet-derived growth factor receptor (PDGFRa) ${ }^{+}$stromal cells isolated from the kidneys of 11.5-day-old embryonic mice. This kidney organoid underwent branching morphogenesis and gained higher-order architecture that resembled the embryonic kidney with all segments of nephrons and a contiguous collecting duct system (Taguchi and Nishinakamura 2017). In another study, Xinaris and colleagues examined whether human amniotic fluid stem cells (hAFSCs) could promote

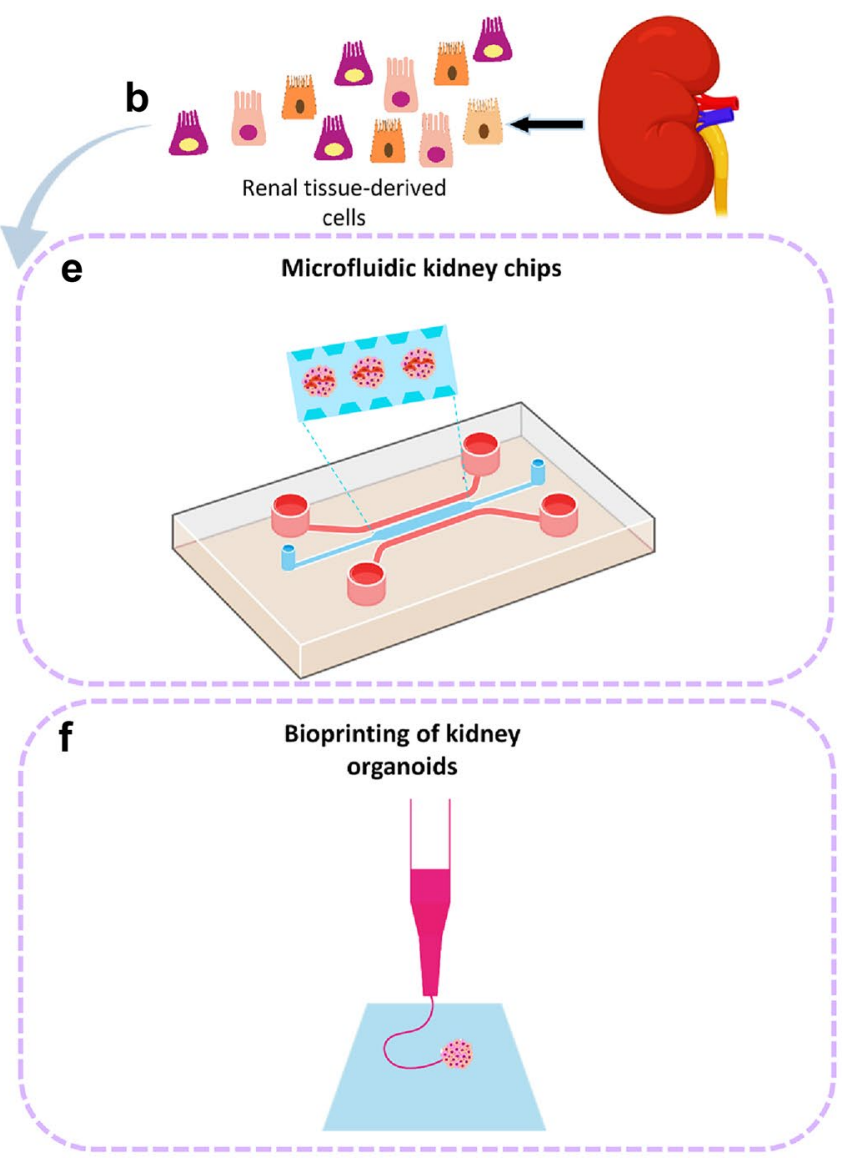

cells or adult stem cells (ASCs) where different approaches increase the complexity of these kidney organoids; (C) co-culture systems; (D) organoid transplantation into vascularized sites such as the subrenal capsule and chorioallantoic membrane (CAM); (E) microfluidic chips; and (F) bioprinting technology

vascularization and maturation of kidney structures. They developed a 3D chimeric organoid by re-aggregating mouse embryonic kidney-derived cells with glial cell-derived neurotrophic factor (GDNF)-expressing hAFSCs at a 10:1 ratio, respectively. GDNF is a key growth factor that is broadly expressed in the MM. GDNF${ }^{+}$cells have better incorporation into developing kidney construction. After 5 days of incubation, they transplanted chimeric kidney organoids under the renal capsules of rats. At 2 weeks after transplantation, human cells contributed to the generation of highly mature tubular structures and vascularized glomeruli, which were significantly associated with the host vascular structures. These chimeric organoids showed kidney-specific functions of the living organism (Xinaris et al. 2016).

The results of several studies indicate that incorporation of ECs more closely mimics the 3D kidney structures during renal development and disease (Musah et al. 2018; Addario et al. 2020; Yin et al. 2020). Musah and colleagues reported that a co-culture system, which combined hiPSC-derived 
podocytes and human kidney glomerular ECs in a microfluidic device, formed a closely recapitulated functional glomerulus with an endothelialized apparatus. This 3D system mimicked adriamycin-induced glomerular injury and proteinuria (Musah et al. 2018). Another study used a coculture system that combined peritubular capillary ECs and renal proximal tubular epithelial cells in the microfluidic kidney chip. This system was efficient for drug screening and nephrotoxicity assays (Yin et al. 2020). Direct cell-cell interactions and angiogenic growth factors secreted by mesenchymal stem cells (MSCs) improved the stabilization and maturation of ECs (Boyd et al. 2011; Paul et al. 2013; Asai et al. 2017). MSCs also have pericyte-like properties and are required for EC stabilization and maturation (Paul et al. 2013). Therefore, some researchers used a co-culture system that combined ECs and MSCs to promote vascularization and maturation of various 3D organotypic cultures (Zahmatkesh et al. 2021). Myosin IIA, which is widely produced by MSCs, provoked a self-condensation process via dynamic movements of the cells (Takebe et al. 2015). Yang et al. established hybrid 3D spheroids using co-culture of immortalized podocytes, human MSCs, and HUVECs at a 1:1:1 ratio, which recapitulated the native glomerulus-like condition. Transplantation of these hybrid cell spheroids into the renal cortex improved engraftment efficiency and kidney function (Yang et al. 2021). In another study, Takebe and colleagues used a tri-culture system by combining heterogeneous cell populations derived from E13.5 mouse kidneys with bone marrow-derived MSCs and HUVECs (cell ratio 10:2:1, respectively). Implantation of kidney organ buds inside the cranium further promoted neovascularization and maturation. Generation of microvascular structures by HUVECs prior to transplantation improved anastomosis with the host vasculature. After 28 days, organ buds gained kidney structures that included some segments of the nephrons such as proximal tubules and glomerular-like structures that contained a vascular network. These 3D kidney structures resembled an immature kidney reminiscent of postnatal kidneys compared to the original embryonicderived primary kidney tissue (Takebe et al. 2015). Moreover, our group established an organotypic co-culture system that generated hPSC-derived renal microtissues. First, we used a slightly modified version of the Morizane and colleagues differentiation protocol to induce nephrogenic IM. These nephrogenic intermediate mesoderm (IM) cells were co-cultured with HUVECs and MSCs at the ratio of 10:7:2, respectively. After 11 days of co-culture, there were 3D human renal organoids that expressed glomerular and renal tubular markers in the culture. Therefore, the addition of endothelial and mesenchymal cells improved the maturation of the renal microtissues (Khoshdel-rad et al. 2021). Co-culture systems that included various cell types and the ECM could provide the highly physiologically relevant systems that improve the complexity of kidney organoids (Zahmatkesh et al. 2021).

\section{Bioprinting of kidney organoids}

Recent advances in 3D bioprinting technology enable the generation of homogeneous, more rapid, highly reproducible kidney organoids (Fransen et al. 2021). Self-organizing kidney organoids have been generated by an extrusion-based $3 \mathrm{D}$ cellular bioprinter from as few as 4000 cells. The generation of 200 kidney organoids took $10 \mathrm{~min}$. In this study, the differentiation protocol reported by Little's laboratory differentiation method was used to produce kidney organoids. On day 7 of differentiation, a 3D bioprinter was used to transfer IM cell aggregates to Transwell filters. This bioprinter could produce highly reproducible large-scale organoids in a short amount of time. The obtained organoids on day 12 were similar in morphology, cell diversity, and gene expression to kidney organoids that were prepared by a manual method. The organoids had various epithelial cells of nephron segments surrounded by a basement membrane. The expressions of glomerular segments (NEPHRIN), proximal tubule (lotus tetragonolobus lectin [LTL], CUBN, CD13), distal tubule/loop of Henle thick ascending limb (ECAD, SLC12A1), and distal connecting segment (transacting T cell-specific transcription factor [GATA]3, ECAD), surrounding stroma (MEIS1/2/3), and endothelial (CD31) markers were observed in these organoids. The organoids generated by automated fabrication provided an opportunity for high content drug screening (Lawlor et al. 2021). In another study, Czerniecki et al. used a liquid handling dispensing system to generate and analyze kidney organoids in microwell arrays. This system allowed high throughput screenings for differentiation, nephrotoxicity, and disease modeling (Czerniecki et al. 2018). Although bioprinting of kidney organoids is an advancing technology, it is still in its infancy (Fransen et al. 2021).

\section{Kidney organoid-on-chips}

Fluid flow is a mechanical force that plays an important role in tissue morphogenesis and provides a niche that more closely represents the in vivo kidney (Ghaffari et al. 2015; Vining and Mooney 2017). Fluid flow in the lumens of kidneys triggers activation of mechanosensitive pathways via microvilli and primary cilia on the apical surfaces of renal tubular cells (Raghavan et al. 2014) Microfluidic kidney chips provide physiological shear stress and interaction between various cell types, and it more accurately reconstitutes a nephrogenic niche. According to the results of some studies, renal cells in the microfluidic device under physiological fluid flow and shear stress have a more sophisticated phenotype. These structures recapitulate functional nephron 
with an endothelialized apparatus (Musah et al. 2018; Yin et al. 2020). In 2019, a study was conducted at Harvard University on the role of fluid shear stress in vascularization and maturation of kidney organoids. The researchers placed hPSC-derived pre-tubular aggregations on a 1-mm-thick layer of gelatin-fibrin ECM within 3D printed microfluidic chips. The results showed significantly increased populations of $\mathrm{KDR}^{+}$EPCs and melanoma cell adhesion molecule $(\mathrm{MCAM})^{+} /$platelet/endothelial cell adhesion molecule-1 $(\text { PECAM1 })^{+}$ECs within the organoids on day 21 of differentiation under high shear stress $\left(0.008-0.035 \mathrm{dyn} / \mathrm{cm}^{2}\right)$. Also, intravascular anastomoses were found between adjacent organoids on the microfluidic chips. As the organoids developed, the number of PDGFR $\beta$ + pericyte-like cells significantly elevated and migrated around the blood vessels. Cellular communication between endothelial and epithelial cells improved maturation of the tubular and glomerular cells in these kidney organoids (Homan et al. 2019). In another study, Schutgens and colleagues established a microfluidic kidney-on-a-chip that had separate compartments for gel and medium perfusion. Primary renal epithelial cell-derived tubuloids were trypsinized, made into a single-cell suspension, and processed by an OrganoPlate microfluidic system. A confluent tubular layer with leak-tight and polarized tubular structures formed 7 days after seeding. This system allowed fluid flow and interaction between various cell types and it more accurately recapitulated the microenvironment of native kidney tissue. They used this microfluidic system to study active epithelial transport reabsorption of solutes between tubular and ECs (Schutgens et al. 2019).

\section{Role of the extracellular matrix in kidney organoid formation}

During organogenesis, the behavior of different cell populations is regulated by the effects of the microenvironmental signals (Zoldan et al. 2011). The kidney-specific ECM is composed of a complex network of collagen, elastin, proteoglycans, glycoproteins, and growth factors that collectively form the basement membrane and interstitial space of renal cells. These dynamic structures provide mechanical support to the renal cells and regulate the differentiation of neighboring cells. Therefore, they have a critical role in kidney development and physiological function (Bülow and Boor 2019). Mechanosensitive cell surface receptors, such as integrins, sense the biophysical properties of their surrounding ECM (Akkerman and Defize 2017). Furthermore, the biomechanical properties of the ECM play an important role in cell fate determination. Stiffness of the ECM regulates self-organization and differentiation into each embryonic germ layer (Zoldan et al. 2011). The results of studies have shown that the utility of kidney-derived ECM increased the viability, proliferation, and maturation of in vitro kidney structures (Sambi et al. 2017; Su et al. 2018; Guo et al. 2020). K-ECM has a wide variety of cytokines and growth factors, including BDNF, bFGF, bone morphogenetic protein (BMP) 4/5/7, EGF, FGF7, VEGF, and TGF$\beta 1$ (Ding et al. 2020; Guo et al. 2020). Guo and colleagues reported that porcine-derived K-ECM increased the differentiation potential of multipotent human USCs into tubularlike structures within the 3D kidney organoids (Guo et al. 2020). In one study, the effect of ECM stiffness was investigated on self-organization and cell density in a 3D Matrigel culture. The results showed that soft environmental conditions (about 10-20 kPa) increased self-organization and cell condensation (Takebe et al. 2015). Evaluation of the effect of ECM softness on differentiation efficiency into kidney lineage showed that the use of a soft polyacrylamide hydrogel substrate (about $1 \mathrm{kPa}$ ) or soft substrates with mechanical properties similar to an early embryonic niche, such as chick embryo CAM, increased the expressions of mesoderm and renal-specific genes. These organoids had more mature structures compared to organoids cultured on rigid substrates (Garreta et al. 2019; Khoshdel-rad et al. 2021). Geuens and colleagues designed a soft thiol-ene cross-linked alginate hydrogel to encapsulate kidney organoids during $3 \mathrm{D}$ culture, which resulted in reducing the fibrosis markers (collagen 1a1, fibronectin, and $\alpha$-smooth muscle actin). Interestingly, no morphological changes in the renal structures were observed. The results of this study showed that encapsulation of kidney organoids into a soft biomaterial could modulate the organoids' ECM composition and more closely mimic the in vivo microenvironment of the developing kidney (Geuens et al. 2021).

\section{Applications}

\section{Disease modeling}

Kidney organoids provide an excellent platform for disease modeling. In this section, we will discuss the uses of the kidney organoids to model several kidney diseases include polycystic kidney disease (PKD), congenital nephrotic syndrome, renal cell carcinoma (RCC), Wilms' tumor, cystic fibrosis, and viral infections. PKD is a genetic disease that causes numerous fluid-filled cysts to grow in kidney tissues. Autosomal dominant polycystic kidney disease (ADPKD) is caused by mutations in the $P K D 1$ and $P K D 2$ genes. These genes encode the protein channels of polycystins 1 and 2 . In this disease, cysts that form in the tubular epithelium of the kidney lead to massive kidney enlargement, impaired function, and end-stage kidney disease (ESkD) (Jin et al. 2016). Autosomal recessive polycystic kidney disease (ARPKD) is generally the early-onset form of PKD, but it can also manifest during adulthood (Burgmaier et al. 2019). ARPKD is 
predominantly caused by mutations in the PKHDl gene. This gene encodes fibrocystin, a ciliary protein. These patients harbor compound heterozygous mutations within the PKHD1 locus (Bergmann et al. 2003). Furthermore, one study showed that DAZ interacting protein 1-like (DZIPIL), which encodes a ciliary transition zone protein, is the second gene involved in ARPKD pathogenesis (Lu et al. 2017). The main obstacle to the diagnosis of a PKD pathophysiology is the lack of a human cellular model to properly simulate cystogenesis. Freedman et al. cultured hiPSCs in a Matrigel sandwich system to generate pluripotent spheroids. Subsequent activation of the canonical pathway of beta-catenin by the small molecule CHIR99021 for 1.5 days and B27 supplemented media for 12 days directed differentiation of the spheroids into 3D nephron-like structures. These structures contained cell populations with characteristics of proximal tubules, podocytes, stroma, and immature endothelium. Ultrastructural examination showed the absence of foot processes in podocytes and brush border in tubular cells. These nephron-like organoids were composed of a large percentage of non-renal cells, which included ectoderm and lateral mesoderm derivatives. However, the resultant renal organoid was functionally capable of modeling a nephrotoxic kidney epithelial injury and also showed the diseased phenotypes after CRISPR/Cas9 knockout of the PODXL and PKDI/2 genes (Freedman et al. 2015). These researchers conducted another study in 2017 with the same protocol to produce organoids from an hPSC line with mutations in the PKD1 and $P K D 2$ genes. The cells were initially cultured for 21 days in order to differentiate into kidney organoids with fluid-filled cysts. However, the efficiency of cyst formation was very low $(7 \%)$. In another experiment, the organoids were transferred to a suspension culture on day 21. Replating of these organoids to anti-adhesive hydrogel coated plates resulted in a tenfold increase in cyst size. In long-term culture, some of the cysts were more than $1 \mathrm{~cm}$ in diameter. Thus, this organoid model showed that adherent forces play an important role in limiting the deformation of tubular structures and cystogenesis. This in vitro model could be used to understand the mechanisms of PKD pathophysiology (Cruz et al. 2017). In the same year, another study was conducted by the same group. First, they used CRISPR/Cas9 to delete the $P O D X L$ gene that encodes the podocalyxin-like protein in hPSCs. These gene-edited hPSCs were then differentiated into nephron-like kidney organoids in the same manner as their prior research. Podocytes within the organoids were defective in attachment and adhesion. This results of study indicated that podocalyxin has a key role in the assembly of microvilli and lateral spaces between developing podocytes and maturation of podocytes. Thus, these nephron-like kidney organoids are good models for discovering the mechanisms of glomerular disease (Brooks et al. 2017). In 2018, a fully automated liquid-handling robot was established with the intent to enhance differentiation and analyze kidney organoids in microwell arrays. These researchers used this high throughput screening-compatible system for a nephrotoxicity assay and disease modeling. They evaluated modifiers of PKD and revealed that blebbistatin, an inhibitor of nonmuscle myosin II, significantly increased the diameters and numbers of PKD organoid cysts by $40 \%$ compared to only $10 \%$ in control organoids without PKD mutations. The results of this study indicated that the non-muscle myosin regulatory pathway might be a potential target for PKD therapeutic interventions (Czerniecki et al. 2018).

In several studies, kidney organoid models were designed for use in precision medicine. Low and colleagues generated iPSCs from fibroblasts of an ARPKD patient who had compound heterozygous mutations within the PKHD1 locus. The researchers used the CRISPR-Cas9 gene editing tool to correct this mutation and subsequently generated an isogenic iPSC line. Then, they differentiated patient-derived iPSCs into cystic ARPKD kidney organoids. These iPSC-derived kidney organoids recapitulated cystogenesis upon treatment with either forskolin or 8-bromoadenosine $3^{\prime}, 5^{\prime}$-cyclic monophosphate (8-Br-cAMP). These agents upregulated intracellular cyclic AMP (cAMP) as a hallmark of PKD. In contrast, kidney organoids derived from gene-corrected ARPKD iPSCs showed a phenotype that resembled the wild type. They also evaluated the effects of two inhibitors of cystogenesis, thapsigargin and CFTRinh172, on the ARPKD organoids. The results showed that both agents effectively inhibited ARPKD kidney organoid cyst formation in a dosedependent manner. Therefore, ARPKD organoids could be used to determine the efficacy and safety of candidate drugs prior to their assessment in clinical trials (Low et al. 2019) (Fig. 2). Hale and colleagues generated iPSC-derived kidney organoids from a congenital nephrotic syndrome patient with compound heterozygous NPHS1 mutations and sieved 3D glomeruli from these organoids. A comprehensive transcriptional and proteomic study indicated that these glomeruli organoids expressed podocyte-specific genes, maintained polarized localization, and improved GBM components compared to 2D cultures. GBM of the glomeruli organoids comprised the major matrix components - collagen IV a1/2/3/5/6, collagen XVIII, laminina5b2g1 (LAM-521), nidogen 1 and 2, agrin, and perlecan. The protein levels of collagen IVa3 were very low, and the glomeruli organoids lacked a properly developed GBM compared to the native kidney. Nevertheless, these organoids provided a useful platform for the in vitro modeling of human podocytopathies and podocyte toxicity assays (Howden et al. 2018). Inherited mutations are a potential risk factor for RCC and represent a major challenge because there are no $3 \mathrm{D}$ in vitro models to study cancer development. The $c$-met-mutated papillary renal cell carcinoma (PRCC) is a major form of hereditary kidney cancer. In order to design an in vitro 


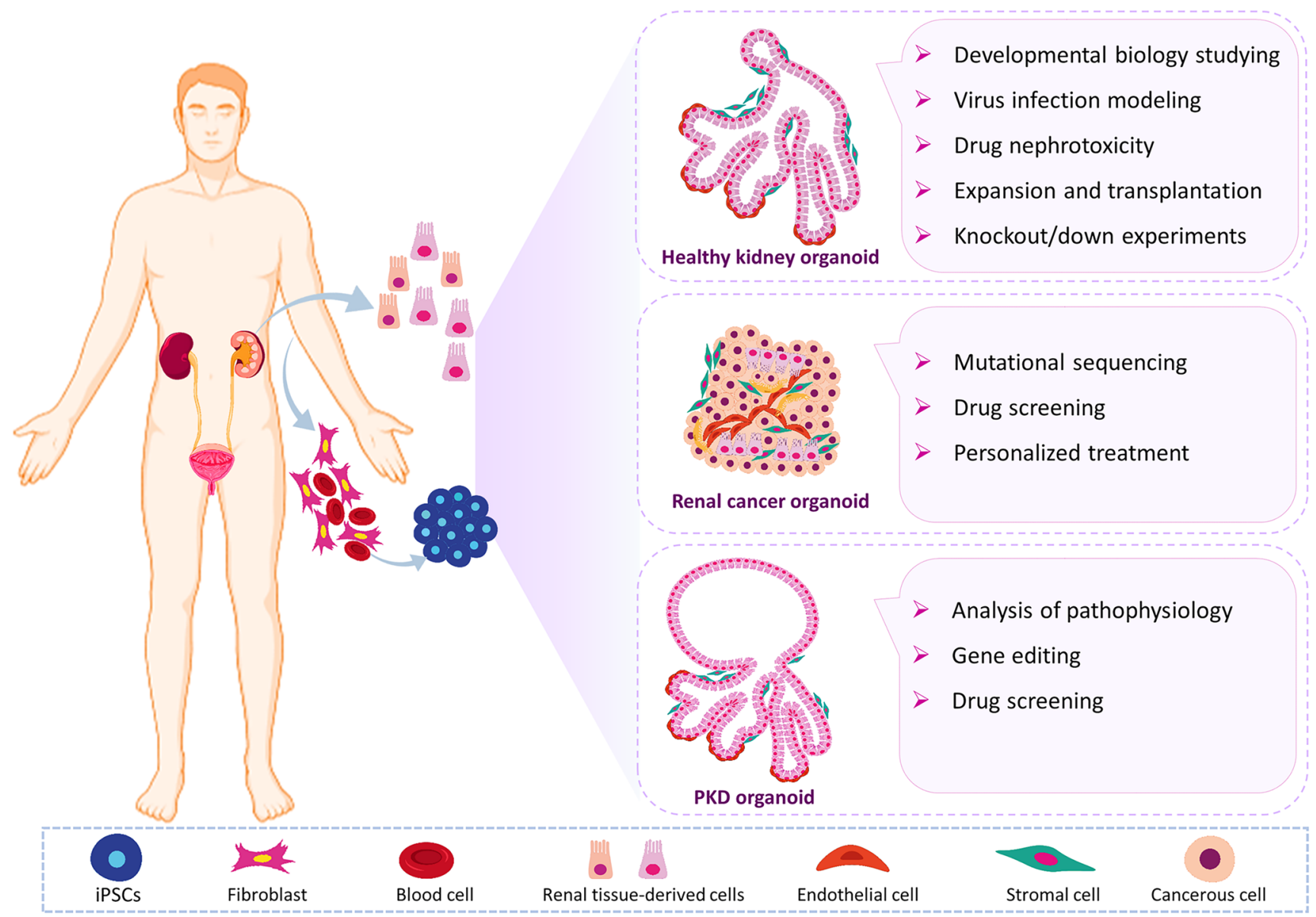

Fig. 2 Potential applications of kidney organoid technology. This technology may rely on the generation of three-dimensional (3D) kidney structures from renal tissue-derived cells (ASCs) or pluripotent stem cells (PSCs). Somatic cells, which include dermal fibroblasts and blood cells, are used to generate induced pluripotent stem cells (iPSCs). Healthy kidney organoids can be exploited to study kidney development, viral infections (BK and SARS-CoV-2), drug nephrotoxicity (cisplatin, gentamicin, adriamycin, etc.), knockout/down of disease-causing mutations with CRISPR/Cas9, and for transplantation into patients. Renal cancer organoids generated from Wilms tumor and renal cell carcinoma patient-derived cells could be used for comprehensive sequencing analysis, drug screening, and personalized treatment. Organoids that are disease models (such as PKD organoids) can be used to study pathophysiology and gene editing and for drug screening

tumors compared to the controls. These organoids displayed an aberrant gene expression signature that was highly associated with the expression pattern found in a large cohort of PRCC patient samples. The researchers identified $11 \mathrm{com}-$ mon genes, including BHLHE4O and $K D M 4 C$, which are factors involved in PRCC pathogenesis (Hwang et al. 2019).

Schutgens et al. isolated ASCs from the urine of patients with a BK virus infection, Wilms' tumor, and cystic fibrosis (CF). They generated ASC-derived kidney tubuloids for the BK virus infection. Then, the tubuloids were treated with BK virus that was isolated from the urine of an immunocompromised patient. BK virus infections are the cause of $5-10 \%$ of transplant rejections in donated kidney recipients. BK virus expanded stably in the human kidney tubuloids, and the virus particles were detected in the culture supernatant. Cidofovir (CDV), an inhibitor of DNA polymerase), ney organoids derived from c-met-iPSCs formed larger 
significantly decreased the number of BK copies. Therefore, the use of tubuloids can mimic clinical histopathology and allow the investigation of the effects of the BK virus on an individual-based nephrotic injury. For CF modeling, the researchers isolated cells from the urine of a patient with $\mathrm{CF}$ and generated 3D renal tubuloids. These tubuloids can originate either from kidney epithelium cells where they stain positive for paired box protein (PAX) 8 or from urothelial cells where they stain positive for P63 and negative for PAX8 in the urine of patients. Urine-derived renal tubuloids were used to investigate the effects of some agents on various $C F$ phenotypes. In another experiment, these researchers established tubuloids from Wilms tumor tissue. Wilms tumor or nephroblastoma is the most common pediatric tumor that accounts for $5 \%$ of childhood malignancies. The cause of this disease might be related to mutations in specific genes of NPCs (Wilms tumor 1 [WT1], catenin beta 1 [CTNNB1], or sine oculis-related homeobox 2 [SIX2]) during kidney development. The tubuloid obtained from the kidney of a patient with Wilms tumor includes three sections: stroma, blastoma, and epithelium, which are present in patients with one of the types of Wilms tumors. Tubuloids derived from these patients express the SIX2 marker, which is specific to stem cells in the developing kidney (Schutgens et al. 2019). Collectively, kidney organoids provide new avenues to study disease pathogenesis and for personalized drug screening (Fig. 2).

\section{Drug screening}

Nephron injury following medication is one of the most common side effects of drugs and their metabolites. The kidneys are highly vascularized organs that receive approximately $20-25 \%$ of the cardiac output (Koning et al. 2020). Toxins in the systemic circulation may accumulate in the vascular, glomerular, and interstitial spaces of kidneys. During pharmaceutical manufacturing processes, $19 \%$ of drugs fail in the third phase of clinical trials because of nephrotoxicity, and this places a heavy economic burden on these companies (Su et al. 2014; Takebe and Taniguchi 2014). Consequently, there is an urgent need for personalized models for drug nephrotoxicity. One adverse effect of medication is acute kidney damage. Kidney organoids provide a platform for drug screening during the drug discovery process by recapitulating kidney tissue in vitro and lead to the identification of candidate drugs, reduce drug development costs, and reduce the rate of acute renal-induced damage (Morizane et al. 2015; Takasato et al. 2015).

Researchers have evaluated organoid function as an experimental model by examining the effect of nephrotoxic drugs such as cisplatin. Cisplatin is a chemotherapy medication used to treat various cancers. Administration of this drug in $35 \%$ of patients causes acute kidney injury (AKI) and apoptosis in renal tubular cells. It has been reported that the concentration of this drug in renal tubular cells is fivefold higher than in blood. The organic cation transporter channel (OCT2) in the basolateral membrane of proximal tubular cells is responsible for uptake of cisplatin into cells. Gamma-glutamyl transpeptidase (GGT) is an enzyme that is expressed in the renal proximal tubular brush borders. The presence of this enzyme is a factor in converting cisplatin to a nephrotoxic agent. In many studies, cisplatin was used to model AKI in kidney organoids (Sprowl et al. 2013). After renal organoid treatment with cisplatin for 24-48 h, increased renal damage was shown as an increase in the expression of specific markers of renal tubular injury and apoptosis (Morizane et al. 2015; Takasato et al. 2015; Ding et al. 2020).

Gentamicin is an antibiotic that damages to nephrons. In several studies, $5 \mathrm{mg} / \mathrm{ml}$ gentamicin was administered for $48 \mathrm{~h}$ in order to damage proximal tubular cells in kidney organoids. The results showed that this drug increased the expressions of markers of kidney damage in the proximal tubules (Morizane et al. 2015; Hiratsuka et al. 2019; Khoshdel-rad et al. 2021). Ding and colleagues assessed the potential for primary renal cell-derived renal organoids as a nephrotoxicity assay. They used a high dose $(200 \mathrm{mM})$ of aspirin and penicillin $\mathrm{G}$ for 2 days. These drugs are widely used in clinical practice and either a high dose or their long-term consumption adversely affects kidney function. The data demonstrated that treatment with aspirin and penicillin $\mathrm{G}$ increased mRNA levels of KIM1 in cells in the renal organoids. GGT activity in drug-treated organoids was remarkably lower than the control group. This study indicated that primary renal cell-derived renal organoids could be a promising tool for drug nephrotoxicity testing (Ding et al. 2020).

Kidney organoids can be suitable platforms to evaluate the effects of drugs on kidney disease. In 2020, coinciding with the COVID-19 pandemic, Monteil et al. investigated the therapeutic effects of human recombinant soluble ACE2 (hrsACE2) on severe acute respiratory syndrome coronavirus (SARS-CoV)-2-infected kidney organoids. They differentiated hESCs into 3D kidney organoids, as previously reported (Garreta et al. 2019). These 3D kidney structures contained $\mathrm{LTL}^{+} / \mathrm{SCL} 3 \mathrm{~A} 1^{+} / \mathrm{SCL} 27 \mathrm{~A} 2^{+} / \mathrm{SCL} 5 \mathrm{~A} 12^{+}$proximal tubules and $\mathrm{PODXL}^{+} / \mathrm{NPHS}^{+} / \mathrm{NPHS}^{+}$podocytes. These two cell types in the kidney organoids expressed angiotensin-converting enzyme 2 (ACE2), a major receptor for SARS-CoV. The expression pattern of ACE2 within the kidney organoids was similar to normal kidney tissue (Monteil et al. 2020). Therefore, SARS-CoV-2 could replicate in the kidney organoids and directly infect these 3D structures. This research group showed that hrsACE-2 significantly inhibited SARS-CoV-2 attachment to cells during the early stages of infection. Accordingly, kidney 
organoids can be a useful model to study SARS-CoV-2 infection (Monteil et al. 2020; Shpichka et al. 2020).

\section{Personalized medicine}

A major goal of personalized medicine is phenotypic correction by direct restoration, elimination, or repair of mutated genes in patients. Kidney organoids have been used to assess a new genetic mutation involved in the development of kidney disease. Mutations in the retrograde intraflagellar transport 140 (IFT140) gene lead to nephronophilia. This gene encodes one of the subunits of the IFT-A complex that plays a critical role in retrograde intraflagellar transport (IFT). The renal organoids that carry this causative mutation have shorter tubules and shortened, club-shaped primary cilia. Transcriptomic analysis of these organoids indicated decreased expressions of genes involved in the cytoskeletal organization within the cell, cell-junction integrity, and apical-basal polarity. Defects in these processes led to the formation of cysts in Matrigel. In patient-derived iPSCs, the mutated gene was corrected with a clustered regularly interspaced short palindromic repeat (CRISPR)/Cas9 system, and the defective phenotype in iPSC-derived renal organoids was rescued (Forbes et al. 2018).

A number of genetic mutations affect kidney function by attacking glomeruli, the microscopic filters of nephrons. In the glomerulus, the podocytes (glomerular epithelial cells) play an important role in the filtration process. Podocytes are associated with cytoplasmic extensions called foot processes. The slit diaphragm between neighboring foot processes prevents the passage of large proteins into urine. Nephrin and podocin are major components of the slit diaphragm proteins, which are encoded by the NPHSI and NPHS2 genes, respectively. Mutations in the NPHS1 gene lead to congenital nephrotic syndrome, an autosomal recessive disease that manifests as the presence of proteins in the urine (proteinuria) (Heeringa et al. 2008). In a study, kidney organoids were generated from iPSC-derived from a patient with an NPHS1 missense mutation. The mutant nephrin was unable to be located on the surface of the podocytes within the organoid structures. After transplantation, there was impaired slit diaphragm formation in the podocytes. Subsequently, genetic correction of a single amino acid mutation in the NPHS1 gene led to co-localization of nephrin and other slit diaphragm-associated proteins. Therefore, podocytes within the organoid exhibited the normal phenotype and rescued the nephrotic syndrome (Tanigawa et al. 2018).

\section{Regenerative medicine}

Kidney disease is a global public health problem. Diseases such as acute and chronic renal failure, as well as PKD, may lead to ESKD. Conventional treatments include dialysis and kidney transplantation. Lack of donated organs and the need for lifelong use of immunosuppressive drugs are among the main challenges faced by conventional treatments. The goal of regenerative medicine is to replace or regenerate damaged human cells, tissues, and organs in order to completely restore or establish their normal structures and functions (Davies et al. 2020). Organoid technology has given researchers the ability to produce autologous or human lymphocytes antigen-compatible tissues. The formation of blood vessels is an important event in maturation and function of kidney organoids (Low et al. 2019). In several studies, human stem cell-derived kidney organoids were transplanted into animal models. Researchers observed that, after a few days, these organoids could communicate with host blood vessels, without the addition of exogenous substances, and host blood cells were observed in the vessels inside the organoids (Bantounas et al. 2018; Low et al. 2019). The results of a study showed a significant improvement in renal morphogenesis after transplantation of kidney organoids under a rat kidney capsule. These organoids communicated with the host blood vessels, and the researchers observed blood flow in the glomerular structures in addition to formation of the GBM and fenestrated ECs (specific to the kidney glomerular structures) (Bantounas et al. 2018).

Despite the growing use of kidney organoids in basic research, their clinical translation into cell replacement therapies is in its infancy. The safety and efficacy of organoid-based cell therapies require proper cellular composition, appropriate incorporation and vascularization into the host tissues and organs, and the demonstration of functional roles. Nevertheless, it is anticipated that kidney organoids will open avenues for future regenerative therapies (Vives and Batlle-Morera 2020).

\section{Conclusion and perspectives}

There is major progress in our understanding of cellular components and microenvironmental cues of kidney development. Therefore, organoids can be developed by following cellular communications similar to those that occur during embryogenesis. In the future, organoid models combined with recent biotechnological progress such as microfluidics, synthetic ECM, co-culture systems, and 3D bioprinting technology will offer exciting improvements for precise application of this technology. The use of organoid technology for regenerative medicine is still in its infancy, and further studies should be performed to elucidate the signaling pathways involved in maturation, vascularization, and the immune response. Kidney organoids open new perspectives for the discovery of potentially 
novel pathways that are involved in disease pathogenesis and new drugs for future precision medicine strategies.

Acknowledgements We would like to express our sincere appreciation to Dr. Hossein Baharvand and Dr. Stefano Da Sacco for their scientific assistance and critical comments.

Author contribution Niloofar Khoshdel-Rad developed the concept for this article, contributed to the design and preparation of this manuscript, and conducted the literature review and table layout. Amin Ahmadi contributed to the preparation of some part of this manuscript for writing. Reza Moghadasali drafted the manuscript and revised it critically for all content. All authors read and approved the final manuscript.

\section{Declarations}

Conflict of interest The authors declare no competing interests.

\section{References}

Addario G, Djudjaj S, Farè S et al (2020) Microfluidic bioprinting towards a renal in vitro model. Bioprinting. https://doi.org/10. 1016/j.bprint.2020.e00108

Akkerman N, Defize LHK (2017) Prospects \& overviews dawn of the organoid era. Bio Essays 39:1-10. https://doi.org/10.1002/bies. 201600244

Al-Awqati Q (2013) Cell biology of the intercalated cell in the kidney. FEBS Lett 587:1911-1914. https://doi.org/10.1016/j.febslet.2013.05.007

Al-Awqati Q, Oliver JA (2002) Stem cells in the kidney. Kidney Int 61:387-395. https://doi.org/10.1046/j.1523-1755.2002.00164.x

Asai A, Aihara E, Watson C et al (2017) Paracrine signals regulate human liver organoid maturation from induced pluripotent stem cells. Development 144(6):1056-1064. https://doi.org/10.1242/ dev. 142794

Bantounas I, Ranjzad P, Tengku F et al (2018) Generation of functioning nephrons by implanting human pluripotent stem cell-derived kidney progenitors. Stem Cell Reports 10:766-779. https://doi. org/10.1016/j.stemcr.2018.01.008

Bergmann C, Senderek J, Sedlacek B et al (2003) Spectrum of mutations in the gene for autosomal recessive polycystic kidney disease (ARPKD/PKHD1). J Am Soc Nephrol 14:76-89. https://doi.org/ 10.1097/01.ASN.0000039578.55705.6E

Bohnenpoll T, Bettenhausen E, Weiss AC et al (2013) Tbx18 expression demarcates multipotent precursor populations in the developing urogenital system but is exclusively required within the ureteric mesenchymal lineage to suppress a renal stromal fate. Dev Biol 380:25-36. https://doi.org/10.1016/j.ydbio.2013.04.036

Boyd NL, Nunes SS, Jokinen JD et al (2011) Microvascular mural cell functionality of human embryonic stem cell-derived mesenchymal cells. Tissue Eng A 17:1537-1548. https://doi.org/10.1089/ten. tea.2010.0397

Bronner-Fraser M, Fraser S (1988) Cell lineage analysis reveals multipotency of some avian neural crest cells. Nature 336:403-405. https://doi.org/10.1038/335161a0

Brooks CR, McNagny KM, Liu Y et al (2017) Gene-edited human kidney organoids reveal mechanisms of disease in podocyte development. Stem Cells 35:2366-2378. https://doi.org/10.1002/stem.2707

Bülow RD, Boor P (2019) Extracellular matrix in kidney fibrosis : more than just a scaffold. J Histochem Cytochem 67:643-661. https://doi.org/10.1369/0022155419849388
Burgmaier K, Kilian S, Bammens B et al (2019) Clinical courses and complications of young adults with autosomal recessive polycystic kidney disease (ARPKD). Sci Rep 9:1-11. https://doi.org/10. 1038/s41598-019-43488-w

Chow T, Wong FTM, Monetti C et al (2020) Recapitulating kidney development in vitro by priming and differentiating mouse embryonic stem cells in monolayers. NPJ Regen Med. https://doi.org/10. 1038/s41536-020-0092-5

Cruz NM, Song X, Czerniecki SM et al (2017) Organoid cystogenesis reveals a critical role of microenvironment in human polycystic kidney disease. Nat Mater 16:1112-1119. https://doi.org/10.1038/ NMAT4994

Czerniecki SM, Cruz NM, Harder JL et al (2018) High-throughput screening enhances kidney organoid differentiation from human pluripotent stem cells and enables automated multidimensional phenotyping. Cell Stem Cell 22:929-940.e4. https://doi.org/10. 1016/j.stem.2018.04.022

Davies JA, Murray P, Wilm B (2020) Regenerative medicine therapies: lessons from the kidney. Curr Opin Physiol 14:41-47. https://doi. org/10.1016/j.cophys.2019.12.008

Ding B, Sun G, Liu S et al (2020) Three-dimensional renal organoids from whole kidney cells: generation, optimization, and potential application in nephrotoxicology in vitro. Cell Transplant 29:1-10. https://doi.org/10.1177/0963689719897066

Faria J, Ahmed S, Gerritsen KGF et al (2019) Kidney-based in vitro models for drug-induced toxicity testing. Arch Toxicol 93:33973418. https://doi.org/10.1007/s00204-019-02598-0

Forbes TA, Howden SE, Lawlor K et al (2018) Patient-iPSC-derived kidney organoids show functional validation of a ciliopathic renal phenotype and reveal underlying pathogenetic mechanisms. Am J Hum Genet 102:816-831. https://doi.org/10.1016/j.ajhg.2018. 03.014

Francipane MG, Lagasse E (2015) The lymph node as a new site for kidney organogenesis. Stem Cells Transl Med 4:295-307. https:// doi.org/10.5966/sctm.2014-0208

Fransen MFJ, Addario G, Bouten CVC et al (2021) Bioprinting of kidney in vitro models: cells, biomaterials, and manufacturing techniques. Essays Biochem 65:587-602

Freedman BS, Brooks CR, Lam AQ et al (2015) Modelling kidney disease with CRISPR-mutant kidney organoids derived from human pluripotent epiblast spheroids. Nat Commun. https://doi.org/10. 1038/ncomms9715

Garreta E, Prado P, Tarantino C et al (2019) Fine tuning the extracellular environment accelerates the derivation of kidney organoids from human pluripotent stem cells. Nat Mater. https://doi.org/10. 1038/s41563-019-0287-6

Geuens T, Ruiter FAA, Schumacher A et al (2021) Thiol-ene crosslinked alginate hydrogel encapsulation modulates the extracellular matrix of kidney organoids by reducing abnormal type 1a1 collagen deposition. Biomaterials 275:120976. https://doi.org/10. 1016/j.biomaterials.2021.120976

Ghaffari S, Leask RL, Jones EAV (2015) Flow dynamics control the location of sprouting and direct elongation during developmental angiogenesis. Development 142:4151-4157. https://doi.org/10. 1242/dev. 128058

Guillaume R, Bressan M, Herzlinger D (2009) Paraxial mesoderm contributes stromal cells to the developing kidney. Dev Biol 329:169_ 175. https://doi.org/10.1016/j.ydbio.2009.02.034

Guo H, Deng N, Dou L et al (2020) 3-D human renal tubular organoids generated from urine-derived stem cells for nephrotoxicity screening. ACS Biomater Sci Eng. https://doi.org/10.1021/acsbi omaterials.0c01468

Gupta N, Dilmen E, Morizane R (2020) 3D kidney organoids for bench-to-bedside translation. J Mol Med. https://doi.org/10.1007/ s00109-020-01983-y 
Heeringa SF, Vlangos CN, Chernin G et al (2008) Thirteen novel NPHS 1 mutations in a large cohort of children with congenital nephrotic syndrome. Nephrol Dial Transplant 23:3527-3533. https://doi.org/10.1093/ndt/gfn271

Hiratsuka K, Monkawa T, Akiyama T et al (2019) Induction of human pluripotent stem cells into kidney tissues by synthetic mRNAs encoding transcription factors. Sci Rep 9:1-13. https:// doi.org/10.1038/s41598-018-37485-8

Homan KA, Gupta N, Kroll KT et al (2019) Flow-enhanced vascularization and maturation of kidney organoids in vitro. Nat Methods. https://doi.org/10.1038/s41592-019-0325-y

Howden SE, Hale LJ, Oshlack A et al (2018) 3D organoid-derived human glomeruli for personalised podocyte disease modelling and drug screening. Nat Commun. https://doi.org/10.1038/ s41467-018-07594-Z

Hwang JW, Desterke C, Féraud O et al (2019) IPSC-derived cancer organoids recapitulate genomic and phenotypic alterations of c-met-mutated Hereditary Kidney Cancer. bioRxiv. https://doi. org/10.1101/518456

Jin M, Xie Y, Chen Z et al (2016) System analysis of gene mutations and clinical phenotype in Chinese patients with autosomaldominant polycystic kidney disease. Sci Rep 6:1-9. https://doi.org/ 10.1038/srep35945

Kang HM, Lim JH, Noh KH et al (2019) Effective reconstruction of functional organotypic kidney spheroid for in vitro nephrotoxicity studies. Sci Rep 9:1-17. https://doi.org/10.1038/ s41598-019-53855-2

Khoshdel-rad N, Zahmatkesh E, Moeinvaziri F (2021) Promoting maturation of human pluripotent stem cell-derived renal micro-tissue by incorporation of endothelial and. Stem Cells Dev 30:428-440. https://doi.org/10.1089/scd.2020.0189

Khoshdel Rad N, Aghdami N, Moghadasali R (2020) Cellular and molecular mechanisms of kidney development: from the embryo to the kidney organoid. Front Cell Dev Biol 8:1-16. https://doi. org/10.3389/fcell.2020.00183

Kobayashi A, Mugford JW, Krautzberger AM et al (2014) Identification of a multipotent self-renewing stromal progenitor population during mammalian kidney organogenesis. Stem Cell Reports 3:650-662. https://doi.org/10.1016/j.stemcr.2014.08. 008

Koning M, Leuning DG, Vanslambrouck JM et al (2018) Renal subcapsular transplantation of PSC-derived kidney organoids induces neo-vasculogenesis and significant glomerular and tubular maturation in vivo. Stem Cell Reports 10:751-765. https://doi.org/10. 1016/j.stemcr.2018.01.041

Koning M, van den Berg CW, Rabelink TJ (2020) Stem cell-derived kidney organoids: engineering the vasculature. Cell Mol Life Sci 77:2257-2273. https://doi.org/10.1007/s00018-019-03401-0

Kumar SV, Er PX, Lawlor KT et al (2019) Kidney micro-organoids in suspension culture as a scalable source of human pluripotent stem cell-derived kidney cells. Development. https://doi.org/10. $1242 /$ dev. 172361

Lawlor KT, Vanslambrouck JM, Higgins JW et al (2021) Cellular extrusion bioprinting improves kidney organoid reproducibility and conformation. Nat Mater 20:260-271. https://doi.org/10.1038/ s41563-020-00853-9

Liao J, Yu Z, Chen Y et al (2020) Single-cell RNA sequencing of human kidney. Sci Data 7:1-9. https://doi.org/10.1038/ s41597-019-0351-8

Little MH, Combes AN (2019) Kidney organoids: accurate models or fortunate accidents. Genes Dev 33:1319-1345. https://doi.org/10. 1101/gad.329573.119

Low JH, Li P, Guo E et al (2019) Generation of human PSC-derived kidney organoids with patterned nephron segments and a de novo vascular network. Stem Cell 25:1-15. https://doi.org/10.1016/j. stem.2019.06.009
Lu H, Ott E, Kaeslin G et al (2017) Mutations in DZIP1L, which encodes a ciliary transition zone protein, cause autosomal recessive polycystic kidney disease. Nat Genet 49:1025-1034. https:// doi.org/10.1038/ng.3871.Mutations

Miyoshi T, Hiratsuka K, Garcia Saiz E, Morizane R (2019) Kidney organoids in translational medicine: disease modeling and regenerative medicine. Dev Dyn. https://doi.org/10.1002/dvdy.22

Monteil V, Kwon H, Prado P et al (2020) Inhibition of SARS-CoV-2 infections in engineered human tissues using clinical-grade soluble human ACE2. Cell 181:905-913.e7. https://doi.org/10.1016/j. cell.2020.04.004

Morizane R, Lam AQ, Freedman BS et al (2015) Nephron organoids derived from human pluripotent stem cells model kidney development and injury. Nat Biotechnol 33:1193-1200. https://doi.org/ 10.1038/nbt.3392

Mugford JW, Sipilä P, Mcmahon JA, Mcmahon AP (2008) Osr1 expression demarcates a multi-potent population of intermediate mesoderm that undergoes progressive restriction to an Osr1dependent nephron progenitor compartment within the mammalian kidney. Dev Biol 324:88-98. https://doi.org/10.1016/j.ydbio. 2008.09.010.Osr1

Munro DAD, Hohenstein P, Davies JA (2017) Cycles of vascular plexus formation within the nephrogenic zone of the developing mouse kidney. Sci Rep 7:1-13. https://doi.org/10.1038/ s41598-017-03808-4

Musah S, Dimitrakakis N, Camacho DM et al (2018) Directed differentiation of human induced pluripotent stem cells into mature kidney podocytes and establishment of a glomerulus chip. Nat Protoc 13:1662-1685. https://doi.org/10.1038/s41596-018-0007-8

Paul JD, Coulombe KLK, Toth PT et al (2013) SLIT3-ROBO4 activation promotes vascular network formation in human engineered tissue and angiogenesis in vivo. J Mol Cell Cardiol 64:124-131. https://doi.org/10.1016/j.yjmcc.2013.09.005

Raghavan V, Rbaibi Y, Pastor-Soler NM et al (2014) Shear stressdependent regulation of apical endocytosis in renal proximal tubule cells mediated by primary cilia. Proc Natl Acad Sci U S A 111:8506-8511. https://doi.org/10.1073/pnas.1402195111

Sambi M, Chow T, Whiteley J et al (2017) Acellular mouse kidney $\mathrm{ECM}$ can be used as a three-dimensional substrate to test the differentiation potential of embryonic stem cell derived renal progenitors. Stem Cell Rev Reports 13:513-531. https://doi.org/10. 1007/s12015-016-9712-2

Schmidt-ott KM, Yang J, Chen X et al (1993) Novel regulators of kidney development from the tips of the ureteric bud. J Am Soc Nephrol 16:1993-2002. https://doi.org/10.1681/ASN.2004121127

Schnaper HW (2014) Remnant nephron physiology and the progression of chronic kidney disease. Pediatr Nephrol 29:193-202. https:// doi.org/10.1007/s00467-013-2494-8

Schutgens F, Rookmaaker MB, Margaritis T et al (2019) Tubuloids derived from human adult kidney and urine for personalized disease modeling. Nat Biotechnol 37:303-313. https://doi.org/10. 1038/s41587-019-0048-8

Shankar AS, Du Z, Mora HT et al (2021) Human kidney organoids produce functional renin. Kidney Int 99:134-147. https://doi.org/ 10.1016/j.kint.2020.08.008

Shpichka A, Bikmulina P, Peshkova M et al (2020) Engineering a model to study viral infections: bioprinting, microfluidics, and organoids to defeat coronavirus disease 2019 (COVID-19). Int J Bioprinting 6:1-20. https://doi.org/10.18063/IJB.V6I4.302

Sinha S, Saifudeen Z, Hering-Smith KS et al (2017) P63+ ureteric bud tip cells are progenitors of intercalated cells. JCI Insight 2:1-9. https://doi.org/10.1172/jci.insight.89996

Sprowl JA, Van Doorn L, Hu S et al (2013) Conjunctive therapy of cisplatin with the OCT2 inhibitor cimetidine: influence on antitumor efficacy and systemic clearance. Clin Pharmacol Ther 94:585-592. https://doi.org/10.1038/clpt.2013.145 
Su J, Satchell SC, Shah RN, Wertheim JA (2018) Kidney decellularized extracellular matrix hydrogels: rheological characterization and human glomerular endothelial cell response to encapsulation. $\mathrm{J}$ Biomed Mater Res - Part A 106:2448-2462. https://doi.org/10. 1002/jbm.a.36439

Su R, Li Y, Zink D, Loo LH (2014) Supervised prediction of druginduced nephrotoxicity based on interleukin- 6 and -8 expression levels. BMC Bioinformatics 15:1-9. https://doi.org/10.1186/ 1471-2105-15-S16-S16

Taguchi A, Kaku Y, Ohmori T et al (2014) Redefining the in vivo origin of metanephric nephron progenitors enables generation of complex kidney structures from pluripotent stem cells. Cell Stem Cell 14:53-67. https://doi.org/10.1016/j.stem.2013.11.010

Taguchi A, Nishinakamura R (2017) Higher-order kidney organogenesis from pluripotent stem cells. Cell Stem Cell 21:730-746.e6. https://doi.org/10.1016/j.stem.2017.10.011

Takasato M, Er PX, Chiu HS et al (2015) Kidney organoids from human iPS cells contain multiple lineages and model human nephrogenesis. Nature 526:564-568. https://doi.org/10.1038/ nature 15695

Takebe T, Enomura M, Yoshizawa E et al (2015) Vascularized and complex organ buds from diverse tissues via mesenchymal celldriven condensation. Stem Cell 16:556-565. https://doi.org/10. 1016/j.stem.2015.03.004

Takebe T, Taniguchi H (2014) Human iPSC-derived miniature organs: a tool for drug studies. Clin Pharmacol Ther 96:310-313. https:// doi.org/10.1038/clpt.2014.110

Tanigawa S, Islam M, Sharmin S et al (2018) Organoids from nephrotic disease-derived iPSCs identify impaired NEPHRIN localization and slit diaphragm formation in kidney podocytes. Stem Cell Reports 11:727-740. https://doi.org/10.1016/j.stemcr.2018.08.003

van den Berg CW, Ritsma L, Avramut MC et al (2018) Renal subcapsular transplantation of PSC-derived kidney organoids induces neovasculogenesis and significant glomerular and tubular maturation in vivo. Stem Cell Reports 10:751-765. https://doi.org/10.1016/j. stemcr.2018.01.041
Vining KH, Mooney DJ (2017) Mechanical forces direct stem behaviour in development and cell regeneration. Nat Rev Mol Cell Biol 18:728742. https://doi.org/10.1038/nrm.2017.108

Vives J, Batlle-Morera L (2020) The challenge of developing human 3D organoids into medicines. Stem Cell Res Ther 11:1-4. https:// doi.org/10.1186/s13287-020-1586-1

Wu H, Uchimura K, Donnelly EL et al (2018) Comparative analysis and refinement of human PSC-derived kidney organoid differentiation with single-cell transcriptomics. Cell Stem Cell 23:869881.e8. https://doi.org/10.1016/j.stem.2018.10.010

Xinaris C, Benedetti V, Novelli R et al (2016) Functional human podocytes generated in organoids from amniotic fluid stem cells. J Am Soc Nephrol 27:1400-1411. https://doi.org/10.1681/ASN.2015030316

Yang WY, Chen LC, Jhuang YT et al (2021) Injection of hybrid 3D spheroids composed of podocytes, mesenchymal stem cells, and vascular endothelial cells into the renal cortex improves kidney function and replenishes glomerular podocytes. Bioeng Transl Med 6:1-12. https://doi.org/10.1002/btm2.10212

Yin L, Du G, Zhang B et al (2020) Efficient drug screening and nephrotoxicity assessment on co-culture microfluidic kidney chip. Sci Rep 10:1-11. https://doi.org/10.1038/s41598-020-63096-3

Zahmatkesh E, Khoshdel-Rad N, Mirzaei H et al (2021) Evolution of organoid technology: lessons learnt in co-culture systems from developmental biology. Dev Biol 475:37-53. https://doi.org/10. 1016/j.ydbio.2021.03.001

Zeng Z, Huang B, Parvez RK et al (2021) Generation of patterned kidney organoids that recapitulate the adult kidney collecting duct system from expandable ureteric bud progenitors. Nat Commun 12:1-15. https://doi.org/10.1038/s41467-021-23911-5

Zoldan J, Karagiannis ED, Lee CY et al (2011) The influence of scaffold elasticity on germ layer specification of human embryonic stem cells. Biomaterials 32:9612-9621. https://doi.org/10.1016/j. biomaterials.2011.09.012

Publisher's Note Springer Nature remains neutral with regard to jurisdictional claims in published maps and institutional affiliations. 